\title{
Highly conserved motifs in non-coding regions of Sirevirus retrotransposons: the key for their pattern of distribution within and across plants?
}

\author{
Alexandros Bousios ${ }^{* *}$, Nikos Darzentas ${ }^{2}$, Athanasios Tsaftaris ${ }^{2,3}$, Stephen R Pearce
}

\begin{abstract}
Background: Retrotransposons are key players in the evolution of eukaryotic genomes. Moreover, it is now known that some retrotransposon classes, like the abundant and plant-specific Sireviruses, have intriguingly distinctive host preferences. Yet, it is largely unknown if this bias is supported by different genome structures.

Results: We performed sensitive comparative analysis of the genomes of a large set of Ty1/copia retrotransposons. We discovered that Sireviruses are unique among Pseudoviridae in that they constitute an ancient genus characterized by vastly divergent members, which however contain highly conserved motifs in key non-coding regions: multiple polypurine tract (PPT) copies cluster upstream of the $3^{\prime}$ long terminal repeat ( $\left.3^{\prime} L T R\right)$, of which the terminal PPT tethers to a distinctive attachment site and is flanked by a precisely positioned inverted repeat. Their LTRs possess a novel type of repeated motif (RM) defined by its exceptionally high copy number, symmetry and core CGG-CCG signature. These RM boxes form CpG islands and lie a short distance upstream of a conserved promoter region thus hinting towards regulatory functions. Intriguingly, in the envelope-containing Sireviruses additional boxes cluster at the $5^{\prime}$ vicinity of the envelope. The $5^{\prime} \mathrm{LTR} /$ internal domain junction and a polyC-rich integrase signal are also highly conserved domains of the Sirevirus genome.

Conclusions: Our comparative analysis of retrotransposon genomes using advanced in silico methods highlighted the unique genome organization of Sireviruses. Their structure may dictate a life cycle with different regulation and transmission strategy compared to other Pseudoviridae, which may contribute towards their pattern of distribution within and across plants.
\end{abstract}

\section{Background}

Retrotransposons and retroviruses (collectively referred as retroelements) can replicate their genomes via an RNA intermediate and insert the copies into new chromosomal locations of the host organism [1,2]. This 'copy and paste' process has the potential to greatly amplify their abundance, even over short evolutionary timescales, enabling them to become a major component of genomes [3-5]. Unlike retrotransposons, retroviruses have additional coding capacity in the form of an envelope $(E N V)$ gene that allows them to enter the extracellular space and infect other individuals. Retrotransposons lack the $E N V$ gene and cannot escape the cell, however they are free to reinfect their host genome.

\footnotetext{
* Correspondence: alexandros.bousios@gmail.com

'Department of Biology and Environmental Science, School of Life Sciences, University of Sussex, Brighton, UK
}

() 2010 Bousios et al; licensee BioMed Central Ltd. This is an Open Access article distributed under the terms of the Creative Commons Attribution License (http://creativecommons.org/licenses/by/2.0), which permits unrestricted use, distribution, and reproduction in any medium, provided the original work is properly cited.
Long Terminal Repeat (LTR) retrotransposons form the most abundant transposable element type in plants, largely accounting for the vast differences in genome sizes [6]. Small genome plants like Arabidopsis (121 Mbp) and rice (389 Mbp) are sparsely populated by LTR retrotransposons, $5.6 \%$ [7] and $17 \%$ [8] respectively. In contrast, the LTR retrotransposon-derived fraction of medium/large genomes may reach up to $75 \%$ in maize $(2.300 \mathrm{Mbp})[9,10]$ and $70 \%$ in barley (5439 Mbp) [11].

The two main superfamilies of LTR retrotransposons are the Ty1/copia (Pseudoviridae) and Ty3/gypsy (Metaviridae) [12], which differ in the order they package their genes in the coding domains. Both typically contain the gag gene and the pol gene region. gag encodes a capsid protein that forms the virus-like particle (VLP), which houses one or two RNA genomes and the enzymes for the cytoplasmic step of reverse 
transcription. pol encodes the enzymatic proteins required for the production of the DNA copy from the RNA template and the insertion of the new copy in the host genome: an aspartic protease $(A P)$, integrase (INT), reverse transcriptase $(R T)$ and $\mathrm{RNaseH}(R H)$ [13]. LTRs flank the retrotransposon genome and contain the cisacting transcriptional regulators, the promoter and termination transcription points. The cis-acting boxes are often recognition sites of stress-related DNA binding factors (DBFs) and may be organized as arrays of two or three repeated motifs (RM) in tandem [14,15]. A 5', untranslated region (5'UTR) serves as the tether domain between the 5'LTR and gag, while the linker domain connects pol and the 3'LTR. At the junctions of the 5' and 3'LTR with the internal retrotransposon genome reside the primer binding site (PBS) and the polypurine tract (PPT), respectively, that prime cDNA synthesis during reverse transcription [16].

The International Committee on the Taxonomy of Viruses (ICTV) has classified Sireviruses into the Pseudoviridae family [17] together with the Pseudovirus and Hemivirus genera. It is the most recently described genus named after the SIRE1 element from soybean, and as they have colonized only plant species, they were originally named Agroviruses [13]. Sireviruses have putative retroviral properties, since many elements contain an $E N V$-like gene in their linker domain [18], which differentiates them from the other Ty1/ copia genera (herein referred as 'classic' Ty1/copia elements, following the analysis of Havecker et al. 2005). Sireviruses have successfully proliferated within plant genomes, comprising a large proportion of the available Ty1/copia populations [19]. Examples of such elements are OPIE- 2 and PREM-2 with more than 127,000 (fragmented) copies each in the maize genome, of which 3,530 and 4,093 are full length elements respectively [9], and Osr8 that is the second most abundant element in rice [8]. Phylogenetic analysis based on the $R T$ domain revealed that Sireviruses are less diverse than classic Ty1/copia retrotransposons, indicating a possible recent evolutionary origin and colonization of their host genomes [20].

Sireviruses differ in the sequence and genomic organization compared to other elements. Whereas the vast majority of plant retrotransposons encode gag and pol as a single open reading frame (ORF), Sireviruses exhibit variation in respect to their organization. Besides the consensus, many elements have pol in two unusual +1 frameshifts relative to gag, of which the one suggests novel ways of translational recoding for pol expression, involving internal ribosomal entry or a bypass strategy [20]. For the bypass mechanism a highly conserved inverted repeat (IR) at the $g a g / p o l$ boundary may provide a favorable secondary structure at the RNA level.
Sireviruses also have a much larger gag gene compared to classic retrotransposons, which is characterized by a central RNA-binding CCHC zinc knuckle and a downstream predicted coiled-coil domain $[13,18]$. The larger capsid protein interacts with the plant Light Chain 8 protein family, which can bind to cargo like cellular proteins and aid their transportation, suggesting it may facilitate VLP assembly or movement towards the nucleus. So far, the evolutionary history of Sireviruses, their life cycle and why they are so successful in invading plant genomes remains unclear.

In this study, the in depth comparative analysis of Pseudoviridae genomes enabled the identification of several short but highly conserved sequence motifs in the otherwise diverse Sirevirus genome. The motifs are found in key regions of the non-coding genome, regions which are necessary for the activation, reverse transcription and integration of the element, or even its virulence capacity through the expression of the $E N V$ gene. Interestingly, one of the motifs is semi-conserved in classic retrotransposons, implying a global fundamental role of its function. Our results suggest that Sireviruses are an ancient retrotransposon lineage that maintains core domains with absolute or high similarity. These domains appear to attribute novel features to the Sirevirus life cycle and may partially explain their distribution within and across plants.

\section{Results}

\section{Sireviruses represent an ancient Ty1/copia genus}

In order to establish a representative dataset for the comparative sequence analysis of Pseudoviridae, 21 Sireviruses were collected from various monocot and eudicot species including (Table S1 in Additional file 1): the five elements in the ICTV Sirevirus database (OPIE-2, PREM-2, SIRE1-1, ToRTL1, Endovir1-1); retrotransposons previously identified as Sireviruses, such as Osr7, Osr8, Osr9, Osr10 from rice [8], HOPIE from maize [21], Lotus2 from lotus, and one element each from sorghum, medicago and citrus [18,22]; plus Tnd1 from tobacco [23], one Vitis vinifera retrotransposon and the Maximus lineage recently reported by Wicker and Keller (2007) that are re-classified as Sireviruses during this work. The classic Ty1/copia retrotransposon population consisted of 30 elements (25 from plants and five from other organisms), representing the other two genera that make up the Pseudoviridae family: 26 Pseudoviruses and four Hemiviruses (Table S1 in Additional file 1). Phylogenetic analysis based on the $R T$ segment, which includes the $5^{\text {th }}$ to $7^{\text {th }} R T$ conserved domains [24] and continues through $R H$ to the end of pol, separates the Sireviruses in a distinct clade with $100 \%$ bootstrap support (Figure S1 in Additional file 2). The relative shorter branch lengths of the 
Sirevirus clade compared to the other retrotransposons are in broad agreement with previous $R T$ phylogenetic analyses [20], suggesting the recent colonization of their host genomes.

Intriguingly, despite the closer evolutionary relationship than classic elements, the sequence organization of the Sirevirus genome appears to be highly divergent, as much as (or even more than) the genome of classic retrotransposons (Figure 1). In fact, excluding the coding domain, the non-coding regions are more diverse in Sireviruses (Table 1), which suggests that the mutation rates of genome change are similar or even higher in the Sirevirus genus compared to other Pseudoviridae retrotransposons. Consequently, the $R T / R H$-derived recent evolutionary origin of Sireviruses may be plasmatic and the genus may in reality represent an ancient lineage. Overall, the average pairwise identity of the fulllength genome of Sireviruses and classic elements is equally low ( $17 \%$ and $13 \%$ respectively), whilst the Sirevirus linker domain and LTRs are extremely diverse (Table 1). Thus, the highly conserved motifs discussed herein are most likely not the result of general sequence similarity within the Sirevirus genome.

\section{Multiple identical PPTs is a unique feature of the Sirevirus linker domain}

Unsupervised discovery of sequence motifs with the use of the TEIRESIAS pattern discovery algorithm [25] primarily identified the downstream 3' end of the internal domain as the most conserved area of the Sirevirus genome, which corresponds to the region where the PPT is located. Postdiscovery analysis, however, differentiated the area into two discrete sub-regions, the PPT and an IR, that have different characteristics. The first motif is the 8 bp 5'AGGGGGAG-3' sequence, which is found precisely $10 \mathrm{bp}$ upstream of the 3'LTR of all elements. TEIRESIAS detected many identical PPT copies in the linker of every Sirevirus, irrespective of the length of the domain or the presence of the $E N V$-gene (Figure $2 \mathrm{~b}$ and Table 2). On average, four PPTs are found in each linker, preferentially clustered near the 3'LTR junction (83\% within $0.5 \mathrm{~kb}$ from the 3'LTR). The biased multiple PPT topology is exemplified in the $E N V$-containing Sireviruses, where the vast majority of upstream PPTs $(56 / 63,89 \%)$ reside after the $E N V$-gene (Table 2). The presence of the PPTs in the linker domain is not coincidental, as the octamer is rarely found in the rest of the Sirevirus genome (0.3/element).

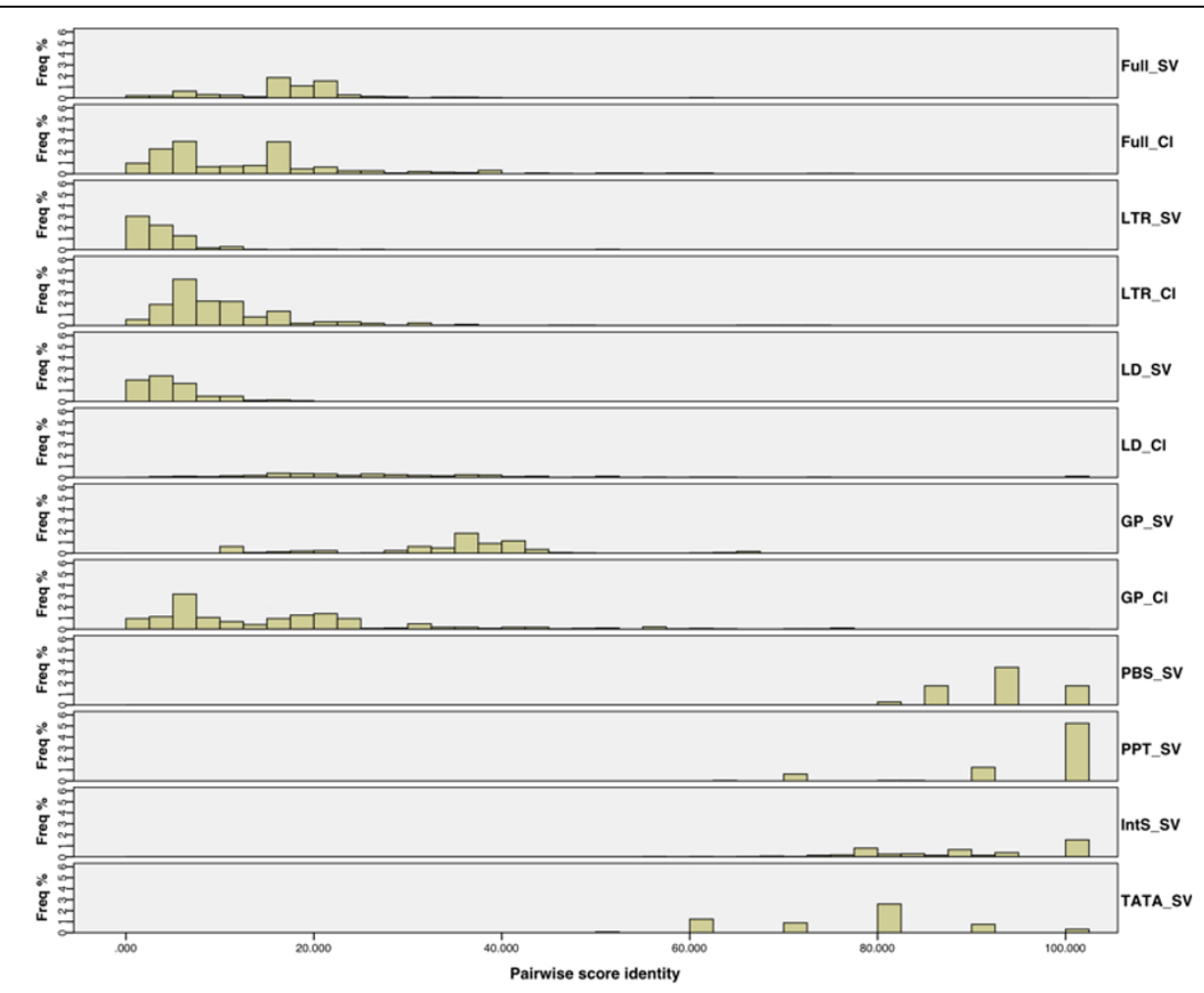

Figure 1 Genome diversity of Ty1/copia retrotransposons. Pairwise score identity of the various genomic regions of Sireviruses and classic elements and of the Sirevirus conserved motifs. Classic Ty1/copia elements with linker domain smaller than 25 bp are not included in the respective analysis. Most domains that are shared between the two retrotransposon datasets appear to be equally divergent (see also Table 1). SV, Sirevirus; Cl, Classic elements; Full, full length element; LD, linker domain; GP, gag-pol; PBS, conserved 5'LTR/internal domain junction; PPT, linker domain/3'LTR junction; IntS, integrase signal; TATA, TATA box-like motif. 
Table 1 Average pairwise score identity (\%) of the various domains of Sirevirus (SV) and classic (Cl) elements, and also of the Sirevirus conserved motifs

\begin{tabular}{lc}
\hline Full length SV - Cl & $16.6-13.2$ \\
LTR SV - Cl & $3.9-10.5$ \\
Linker domain SV - Cl† & $4.8-28.5$ \\
gag/pol SV - Cl & $34.1-16.1$ \\
5'LTR/PBS junction SV $^{\prime}$ & 92.7 \\
PPT/3'LTR junction SV & 95.7 \\
Integrase signal SV & 88.2 \\
TATA box SV & 76.2 \\
\hline
\end{tabular}

† Classic Ty1/copia with linker domain smaller than 25 bp are not included in the respective analysis.

We searched for variants of the 8 bp motif with no significant results, which suggests that sequence integrity is essential and under selection. ToRTL1 and Tnd-1 are the only two Sireviruses that show variation in their PPT nucleotide composition compared to the consensus octamer. Nevertheless, both elements have the terminal G replaced by an A in all their PPTs, which also points towards strong selective pressure instead of random substitutions within the motif.

Conversely to Sireviruses, TEIRESIAS did not discover any conserved motifs in the linker domain and 3'LTR of classic retrotransposons. All elements have a single terminal PPT with a wide range of sequence variation, which does however often resemble the Sirevirus PPT (Figure 2c and Figure 3). Three elements share the Sirevirus octamer, while 17 differ only in one nucleotide. The most common substitutions are the conversions of the $\mathrm{G}$ of the $3^{\text {rd }}$ position to a $\mathrm{T}$ and the $\mathrm{A}$ of the $7^{\text {th }}$ position to a $\mathrm{C}$.

\section{A highly conserved attachment site defines the Sirevirus terminal PPT}

The attachment site consists of a short sequence, usually a di- or trinucleotide, between the PPT and the 3'LTR, and the first few bases, especially the universal dinucleotide TG, of the start of the 3'LTR. It is recognized and processed by INT during the successful insertion of the new copy into the host genome [26]. TEIRESIAS led us to observe that in the Sirevirus 3'LTR junction the conserved motif includes not only the PPT octamer, but also the following four bases. In 20 elements the terminal PPT is followed by the intervening dinucleotide AT, whilst the universal TG indicates the beginning of the 3'LTR, thus generating the Sirevirus 5'-AT/TG-3' attachment site (Figure $2 \mathrm{~b}$ ). The overall nucleotide identity of the combined twelvemer is $95.7 \%$. Interestingly, the sequence following

Table 2 Analysis of the Sirevirus genome size, the position and number of PPTs

\begin{tabular}{|c|c|c|c|c|}
\hline Element & Full/Linker/LTR length $(\mathrm{kb}) \dagger$ & PPTs before $E N V$ & PPTs after $E N V$ & PPTs in linker domain \\
\hline Lotus2 & $12.12 / 1.68 / 1.22$ & - & 4 & 4 \\
\hline Osr10 & $11.97 / 2.04 / 1.56$ & 1 & 3 & 4 \\
\hline Osr9 & $11.63 / 1.68 / 1.51$ & - & 4 & 4 \\
\hline Sorghum & $12.10 / 1.79 / 1.22$ & 1 & 3 & 4 \\
\hline Medicago & $11.93 / 1.14 / 1.27$ & - & 4 & 4 \\
\hline Citrus & $9.98 / 1.28 / 1.16$ & - & 5 & 5 \\
\hline Hopie & $11.87 / 1.37 / 1.68$ & - & 3 & 3 \\
\hline ToRTL1 & $9.68 / 0.88 / 0.80$ & - & 4 & 4 \\
\hline SIRE1-1 & $9.29 / 0.52 / 1.00$ & - & 5 & 5 \\
\hline Endovir1-1 & $9.08 / 1.55 / 0.54$ & - & 3 & 3 \\
\hline Vitis & $9.67 / 1.10 / 1.24$ & 1 & 3 & 4 \\
\hline Barbara & $9.90 / 2.76 / 1.66$ & 1 & 3 & 4 \\
\hline Maximus & $14.42 / 3.96 / 1.41$ & - & 4 & 4 \\
\hline Inga & $12.02 / 1.61 / 1.43$ & 3 & 2 & 5 \\
\hline Usier & $11.77 / 1.84 / 1.57$ & - & 3 & 3 \\
\hline ATCOPIA43 & $9.07 / 1.59 / 0.56$ & - & 3 & 3 \\
\hline Osr7 & $8.92 / 1.31 / 1.62$ & \multicolumn{2}{|c|}{5} & 5 \\
\hline Osr8 & $9.21 / 1.10 / 1.21$ & \multicolumn{2}{|c|}{3} & 3 \\
\hline Tnd-1†† & $8.45 / 0.73 / 1.59$ & \multicolumn{2}{|c|}{4} & 4 \\
\hline OPIE-2 & $8.98 / 0.79 / 1.29$ & \multicolumn{2}{|c|}{4} & 4 \\
\hline PREM-2 & $9.43 / 0.82 / 1.30$ & \multicolumn{2}{|c|}{5} & 5 \\
\hline Avg of Sireviruses & $10.55 / 1.50 / 1.28$ & & & 4/element \\
\hline Avg of classic copia & $5.60 / 0.078 / 0.56$ & \multicolumn{3}{|c|}{ All elements have 1 PPT in their linker } \\
\hline
\end{tabular}

† For elements that contain the ENV-gene, the linker domain length was calculated by combining the segments before and after the ENV-ORF. $+\dagger$ The $5^{\prime} \mathrm{LTR}$ of Tnd- 1 is incomplete and therefore the full length is calculated based on the $3^{\prime} \mathrm{LTR}$. 


\section{A. Genomic organization of Ty1/copia retrotransposons

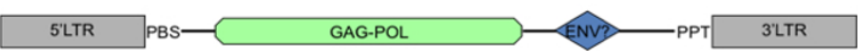

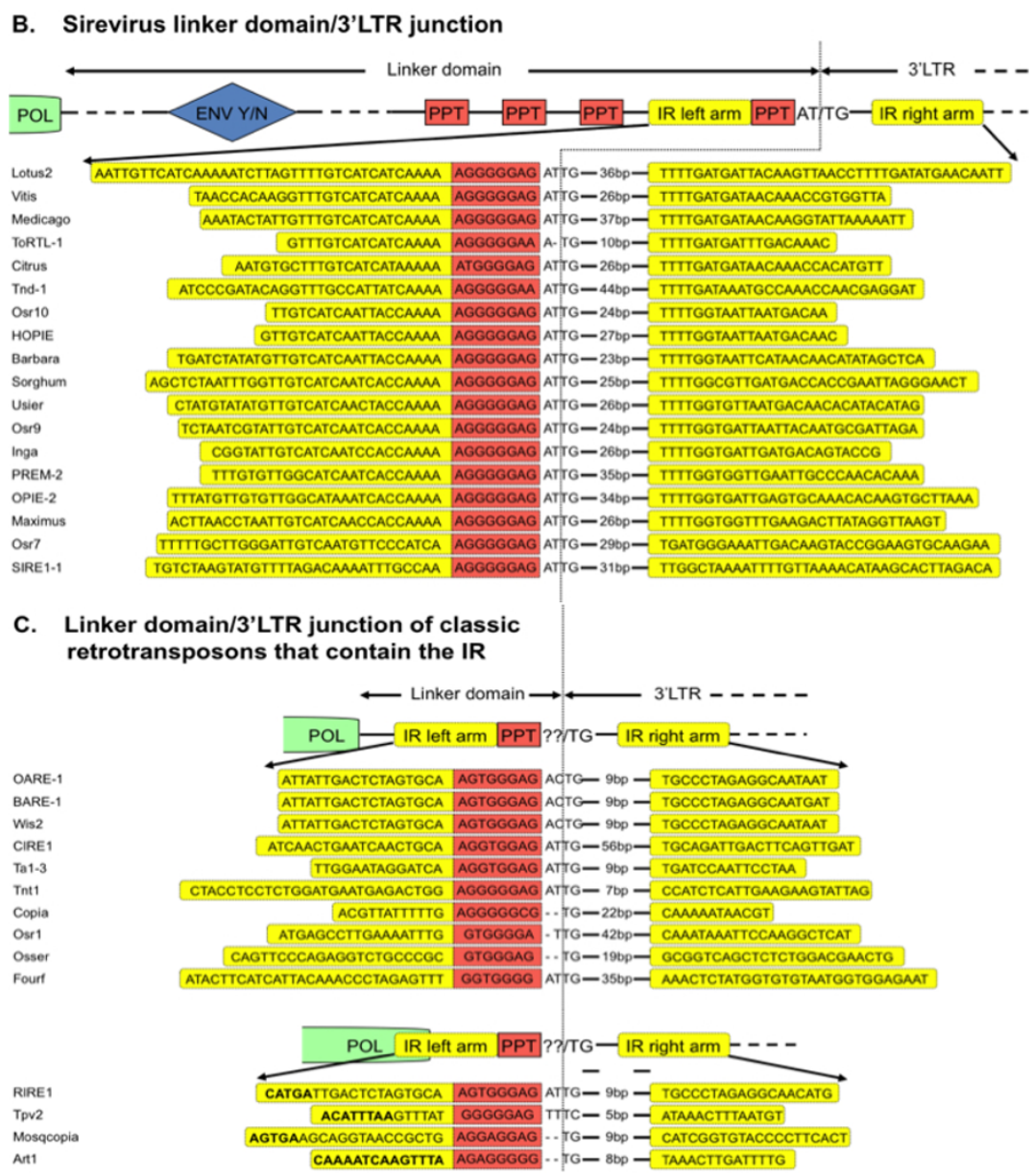

Figure 2 The organization of the linker domain/3'LTR junction of Ty1/copia retrotransposons. (A) Genome structure of Ty1/copia retrotransposons, of which Sireviruses may contain an envelope-like gene. (B, C) Organization of the Sirevirus and classic elements linker domain/3'LTR junction. The IR arms (yellow) surround the loop sequence, of which the terminal PPT octamer (red) occupies the outmost $5^{\prime}$ side. Classic elements with nucleotides in bold indicate the region, where the left arm is located at the $3^{\prime}$ end of the pol gene region, due to the very short linker domain.

the upstream PPTs is highly variable, as only four octamers (out of 63) employ an AT dinucleotide, of which none is linked with the TG motif that would create a putative functional attachment site. In contrast, classic retrotransposons have a highly variable attachment site, with some elements lacking intervening bases between the terminal PPT and the 3'LTR (Figure 2c and Figure 3).

\section{A precisely positioned inverted repeat surrounds the PPT/3'LTR junction of Sireviruses and some classic retrotransposons}

As previously mentioned, TEIRESIAS included bases immediately upstream of the terminal PPT within the most conserved motif of the Sirevirus genome.
Subsequent analysis revealed that this upstream region constitutes the 3' section of a sequence (left IR arm), which is found inverted within the 3'LTR (right IR arm). The IR is present in all but three elements (Osr8, Endovir1-1 and ATCopia43) and shares high nucleotide identity even between Sireviruses that colonize evolutionary distant monocot and eudicot plant species (Figure 2b). The main characteristic of the motif is the precise position of the left arm, which always borders the PPT octamer. The intervening 'loop' sequence is short in length and consists in a $5^{\prime}$ to $3^{\prime}$ direction of the terminal PPT, the 5'-AT/TG-3' attachment site and the beginning of the 3'LTR, which is diverse with large parts often deleted. The right arm starts on average 28 bp inside 


\begin{tabular}{|c|c|}
\hline Element & PPT/3'LTR juncti \\
\hline Sirevirus & AAAAAGGGGGAGATT \\
\hline Tst1 & СTTGAGGGGGAG \\
\hline Tto1 & TGGAAGGGGGAGA \\
\hline Tnt1 & CTGGAGGGGGAGA? \\
\hline Hopscotch & 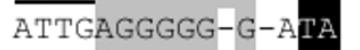 \\
\hline Evelknievel & СTTGAGGGGGCG- \\
\hline Copia & TTTGAGGGGGCG \\
\hline AtRE1 & 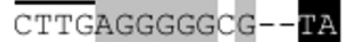 \\
\hline Tgmr & CTTGAGGGGGGG- \\
\hline OARE-1 & TGCAAGTGGGAGACI \\
\hline BARE-1 & TGCAAGTGGGAGAC \\
\hline RIRE1 & 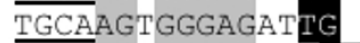 \\
\hline Panzee & 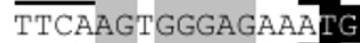 \\
\hline Wis2 & TGCAAGTGGGAGACT \\
\hline Stonor & ACAAAGGGG-AGATT \\
\hline PDR1 & СTTGAGGGG-AG- \\
\hline Mosqcopia & GCTGAGGAGGAG-- \\
\hline Ta1-3 & $\overline{\text { ATCA } A G G T G G A G A T T}$ \\
\hline CIRE1 & TGCAAGGTGGAGATT \\
\hline Tpv2 & $\overline{\text { TTAT }}$-GGGGGAGTTI \\
\hline Melmoth & 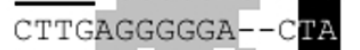 \\
\hline Art1 & TTTAAGAGGGGG- \\
\hline Osser & $\overline{\mathrm{CCGC}}-\mathrm{GTGGGAG}--$ \\
\hline AtRE2 & 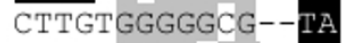 \\
\hline Tca5 & TTGATGGGGAAG--TG \\
\hline Ty 4 & СTTTAAGGG-AGCAT \\
\hline Retrosor 4 & ATTGA-GGG-AGGAT \\
\hline
\end{tabular}

Figure 3 The diversity of the PPT/3'LTR junction of classic Ty1/ copia retrotransposons. Alignment of the conserved Sirevirus PPT/ $3^{\prime}$ LTR junction to the respective region of classic Ty1/copia retrotransposons. Underlined bases represent the 3 ' terminus of the IR left arm, and nucleotides shaded in black indicate the beginning of the $3^{\prime} L T R$. The location of the IR left arm is universally conserved in all Pseudoviridae.

the 3'LTR. Each element contains highly similar arms (on average $89 \%$ identity, 28 bp length), however the sequence conservation is higher in the region surrounding the loop and decreases rapidly towards the periphery of the motif (Figure S2 in Additional file 2). The upstream PPTs lack an IR structure, which suggests that any putative function of the motif is available only through the natural PPT/3'LTR junction.

We discovered that the IR also occurs in almost half classic retrotransposons (Figure 2c), indicating that this structure may serve a fundamental role in the life cycle of the Pseudoviridae. In all cases the left arm extends downstream till the beginning of the predicted PPT, which occupies the 5' border of the loop sequence (Figure 3). This precise arrangement offers great support to the proposed PPT for classic retrotransposons. The IR length within each element is considerably smaller compared to
Sireviruses (on average 88.6\% identity, 19 bp length) (Figure S3 in Additional file 2), whilst the right arm starts on average 18 bp inside the 3'LTR. Characteristically, in elements with very short (RIRE1, Tpv2, Mosqcopia) or absent (Art1) linker the left arm resides partially or completely in the coding domain (nucleotides in bold, Figure 2c).

\section{A novel type of repeated motif (RM) resides in the Sirevirus LTRs and the region upstream of the ENV-gene} We identified in Sireviruses a highly repeated motif with distinctive characteristics in two key areas of the genome of LTR retrotransposons [2], which may indicate its involvement in the regulation of Sirevirus activity. The RM is found in 13 elements and is a Sirevirus specific feature, as it is absent from the genome of classic retrotransposons (Table 3). Besides being abundant in the LTRs, the existence of the $E N V$-gene in nine elements is intriguingly concomitant to the presence of additional RM copies in the linker domain (Table 3, Figure 4b and Figure S4 in Additional file 2). In fact, the distribution of these copies contradicts the multiple PPT topology as the vast majority $(77 / 79,97 \%)$ are preferentially located upstream of the $E N V$-gene (Table 2 and 3). It appears, therefore, that for these copies the $E N V$-gene is the determining factor. Moreover, in Osr10 and HOPIE there are extra copies at the beginning of the $E N V$-gene, near the junction with the linker domain. In contrast, the four Sireviruses without the $E N V$-gene are simultaneously devoid of linker-based copies. The distribution of the RMs in the Sirevirus 3'LTR is also non-random. Most of the copies (117/127, 92\%) cluster within the upper half of each LTR, as they start 200-400 bp after the PPT/3'LTR junction and extend to the following 300-500 bp. As much as 32 units can occur in one element, while all Sireviruses contain at least eight copies of their RM. The overall nucleotide conservation within most elements is remarkable, commonly displaying more than $85 \%$ identity (Table 3 ).

The sequence of all RMs is palindromic with dyad symmetry structure (Table 3), which often characterizes retrotransposon cis-acting transcriptional activators [14]. Besides Tnd-1, the RMs of the other Sireviruses contain a CGG-CCG signature that forms their core structure and generates the palindromy (Figure $4 \mathrm{a}$ ). The highly conserved signature comprises the absolute base of homology, irrespective of the presence of intervening nucleotides between the two symmetrical subunits, or the additional sequence that participates in the symmetry. As an example, Osr10, Osr8, Vitis and Inga share nearly identical RMs, although they have very low sequence homology and reside in evolutionary distant monocot and eudicot species. When we compared the RMs against known plant cis-acting regulatory sequences using the PLACE database [27], we mainly recovered stress-related transcription factor recognition 
Table 3 Sequence, copy number and nucleotide identity of the 13 novel RMs

\begin{tabular}{|c|c|c|c|c|c|c|c|}
\hline \multirow[t]{2}{*}{ Element } & \multirow[t]{2}{*}{ Total copy number } & \multirow[t]{2}{*}{ ENV } & \multicolumn{2}{|c|}{ Linker } & \multirow[t]{2}{*}{ 3'LTR } & \multirow[t]{2}{*}{ Consensus sequencet† } & \multirow[t]{2}{*}{ Nucleotide identity $\%$} \\
\hline & & & bef. ENV & aft. ENV & & & \\
\hline Osr10† & $22(+1)$ & Y & 12 & 0 & 10 & CGGTCTGACCG & 92.7 \\
\hline Osr9 & 20 & Y & 7 & 0 & 13 & TTTTCGGACWTRTCCGAAAA & 88.1 \\
\hline Sorghum & 9 & Y & 4 & 2 & 3 & AGGGCGGCAGTGCCGCCCT & 80.1 \\
\hline Citrus & 12 & Y & 8 & 0 & 4 & AATCCGGCTWRCCGGATT & 66.5 \\
\hline HOPIE† & $21(+4)$ & Y & 7 & 0 & 14 & CGGACCGTCCG & 86.8 \\
\hline Vitis & 12 & Y & 2 & 0 & 10 & CGGTCGACCG & 87.4 \\
\hline Barbara & 26 & Y & 13 & 0 & 13 & TCGGTCYCACCGA & 85.2 \\
\hline Inga & 32 & Y & 18 & 0 & 14 & AGCGGTASTACCGCT & 87.4 \\
\hline Usier & 18 & Y & 6 & 0 & 12 & GTCGGACGTCCGAC & 76.1 \\
\hline OPIE-2 & 9 & $\mathrm{~N}$ & \multicolumn{2}{|c|}{0} & 9 & CACCGGACTGTCCGGTG & 100 \\
\hline PREM-2 & 8 & N & \multicolumn{2}{|c|}{0} & 8 & CACCGGACTGTCCGGTG & 95.5 \\
\hline Osr8 & 9 & N & \multicolumn{2}{|c|}{0} & 9 & CGGTCAGACCG & 85.4 \\
\hline Tnd-1 & 8 & N & \multicolumn{2}{|c|}{0} & 8 & ACAATCGATTGT & 100 \\
\hline
\end{tabular}

† The parenthesis includes the additional copies found at the beginning of the ENV-gene. †† The symmetrical subunits of each RM are underlined, revealing the length and composition of the intervening bases.

sites (Table S2 in Additional file 3). Possibly then, stress-related stimuli may regulate the Sirevirus activation, although experimental confirmation like RM:promoter:GUS fusion analysis is required to correlate the RMs to any regulatory function.

Clusters of RMs in the LTRs and upstream of the ENVgene define the borders of predicted $\mathrm{CpG}$ islands

CpG islands are known to be strongly associated with genes, as they typically occur within or close to their 5 ' terminus near the transcription start site and have been found both in vertebrates [28] and plants [29]. Their unmethylated status has been involved with the regulation of gene expression [30]. The Sirevirus genome seems to contain $\mathrm{CpG}$ islands, which are preferentially found in the LTRs and the region upstream of or within the 5' domain of the $E N V$-gene. Of the 21 elements, Tnd-1, Sorghum and ToRTL1 lack CpG islands, while only twice (in pol of Osr9 and Osr8) does a predicted island occur in a region other than the LTRs or the $E N V$. Among the RM-containing Sireviruses, the RM clusters of HOPIE, OPIE-2, PREM-2, Vitis, Barbara, Inga and Usier are the only CpG islands in their genome, whilst one or two additional islands are present in Osr10, Osr9, Citrus and Osr8 (Figure 4b and Figure S4 in Additional file 2). Presumably, the conserved and high copy number CGG-CCG signature is the key motif of the islands. In most occasions the first and last units
A. Alignment of the RM

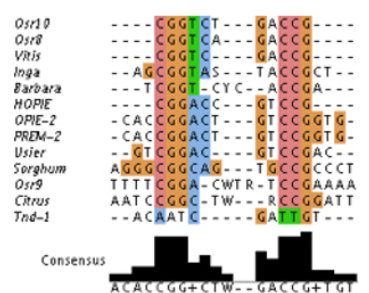

B. Topology of the Sirevirus RM boxes

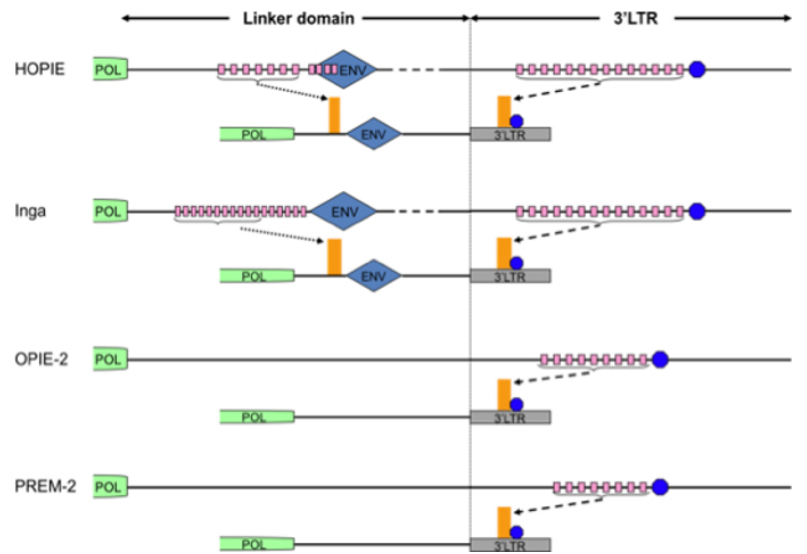

Figure 4 Sequence composition and distribution of the novel Sirevirus RMs. (A) Alignment of the Sirevirus palindromic RM boxes (refer to Table 3 for the intervening bases of each RM), (B) and their organization (pink boxes) in four elements at the $5^{\prime}$ side of the ENV gene (only HOPIE and Inga), and upstream of the TATA box (blue circle) in the $3^{\prime}$ LTR. All or a section of the RM clusters define the borders of CpG islands (orange bar) (all elements are shown in Figure $\$ 4$ in Additional file 2) 
of the RM cluster precisely define the borders of the CpG islands, which by itself supports the hypothesis that they may have a role in the Sirevirus regulatory network. Excluding one very long island in the $E N V$ region of Usier that continues far beyond the last RM, the average 400 bp Sirevirus CpG island starts and finishes approximately $45 \mathrm{bp}$ upstream and downstream of a RM unit, respectively. In contrast to the positional stability of the Sirevirus CpG islands that imply their functionality, most classic elements do not contain a similar pattern (not shown). Only seven have LTR-derived islands, whilst nine lack such a domain and 14 have irregular conformations where CpG islands are present in varied non-LTR locations.

\section{A conserved TATA box lies shortly after the CpG island in the diverse Sirevirus LTR}

Another feature that differentiates Sireviruses from classic retrotransposon genomes is the presence of a conserved TATA box motif in the Sirevirus LTRs. The promoter element 5'-cTATAA/TAT/AAg-3', where lower case letters represent less critical bases [31], resides in all but two elements (Tnd-1 and ATCOPIA43) (Figure $5 \mathrm{a})$ and is approximately located in the middle of each LTR (average nucleotide identity 76\%). Intriguingly, in the RM-containing Sireviruses the TATA box occurs on average $37 \mathrm{bp}$ after the $\mathrm{CpG}$ island, which suggests that the motif corresponds to an authentic promoter and the proximal RM units may regulate its activation (Figure $4 \mathrm{~b}$ and Figure S4 in Additional file 2).

\section{Conservation of the Sirevirus 5'LTR/internal domain junction}

The second most popular sequence motif of the Sirevirus genome according to TEIRESIAS is the 5'LTR/ internal domain junction that accommodates the PBS. The highly conserved upstream junction is divided in three subdomains that in the $5^{\prime}$ to 3 ' direction consists of the universal CA end of the 5'LTR, the intervening sequence and the PBS (Figure $5 \mathrm{~b}$ ). The Sirevirus PBS is only 9 bp (5'-TATCAGAGC-3'), complements the 3' end of the ${ }^{\text {met }}$ tRNA, which is therefore the first primer during reverse transcription. The sharp decrease in sequence conservation after the ninemer implies that Sireviruses paradoxically use only a short segment of the tRNA molecule to prime cDNA synthesis. The intervening sequence is unusually long (5'-ATTGG-3') and in six elements a $G$ replaces the $T$ at the second position. Consequently, the Sirevirus upstream attachment site is the heptamer 5'-CA/ATTGG-3', whilst over the whole length of the 16 bp region the genus maintains $92.7 \%$ identity. In contrast, classic elements contain a more variable 5 'LTR/PBS junction, although and due to the common ${ }^{\text {met }}$ tRNA-derived PBS in many LTR retrotransposons [32], TEIRESIAS discovered a semi-conserved sequence motif in some elements (data not shown).

\section{A polyC-rich integrase singal is located at the $3^{\prime}$ end of the Sirevirus LTRs}

The successful integration of a new retrotransposon copy in the host genome is highly dependent on the interactions between INT and the attachment sites of the element [26]. However, research on retroviruses has shown that binding specificity of INT to the viral DNA is achieved through additional interactions that extend at least $25 \mathrm{bp}$ inwards at the 3' end of the LTR in a domain that is called the 'integrase signal' $[12,33]$. TEIRESIAS identified a conserved motif in this location in 17 Sireviruses. The putative Sirevirus integrase signal is approximately 19 bp long (nucleotide identity $88.2 \%$ ) and contains a distinctive $\mathrm{C}$-rich region in the central domain that varies in size (Figure $5 \mathrm{c}$ ). The average distance between the signal and the LTR end is $21 \mathrm{bp}$. To our knowledge this is the first time such a motif, which also characterizes a whole genus, is reported in the genome of LTR retrotransposons.
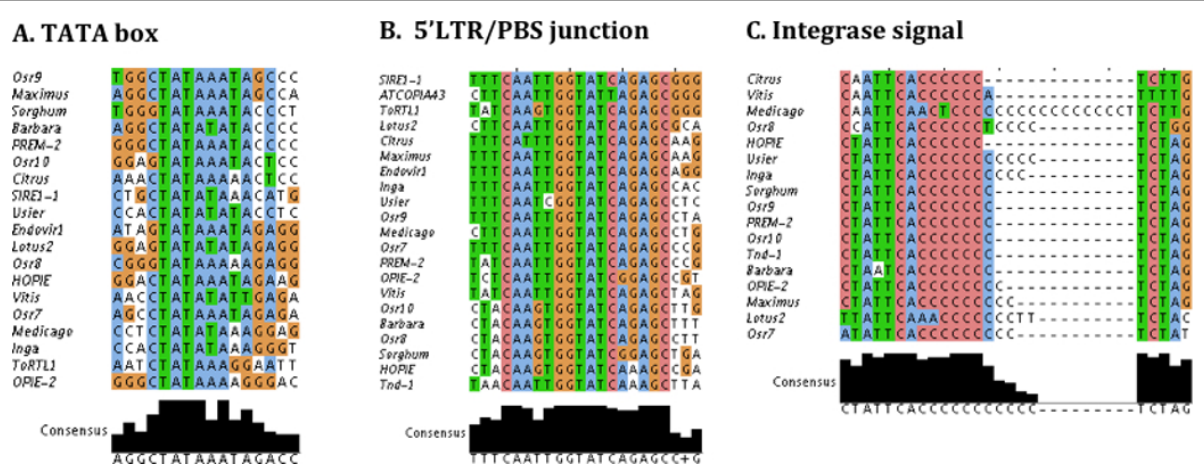

Figure 5 Alignment of the Sirevirus conserved TATA box region (A), 5'LTR/internal domain junction (B), and polyC-rich integrase signal (C). 
Sireviruses are the 'obese' members of the Pseudoviridae family

A comparative analysis of the size between Sireviruses and classic elements revealed that Sireviruses share an exceptionally large genome that clearly differentiates them from other Ty1/copia retrotransposons (Table 2). So far few publications have commented on the length of single Sirevirus elements, the most recent reporting on the large genome of the Maximus lineage [34], ignoring however that Maximus belongs to the Sirevirus genus. The average Sirevirus length $(10.55 \mathrm{~kb})$ is twice as long as that of classic Ty1/copia elements $(5.6 \mathrm{~kb})$. The difference derives from the large LTRs $(1.28 \mathrm{~kb}$ compared to $0.56 \mathrm{~kb})$, linker domain (1.5 kb to $78 \mathrm{bp}$ ), expanded gag (660 to 330 amino acids) and ENV (1.8 kb when present). The sum of the extended regions equals to $5.5 \mathrm{~kb}$ and hence matches the size difference.

\section{Discussion}

The novel in silico analysis of the Sirevirus genome and the comparison to a group of representative classic elements provided what we believe are major insights into the structure of LTR retrotransposons. Our data show that Sireviruses form an ancient lineage, at least as old as those of other classic Ty1/copia elements. The main characteristic of their genome is the high sequence divergence of their large non-coding domains, which are however full of highly conserved microdomains in key regions (Figure 6) that indicate participation in every step of the retrotransposon life cycle, from activation to reverse transcription to integration in the new locus. The position and organization of the motifs do not seem coincidental: i) the novel RMs define CpG islands at the ENV 5' terminus and in the LTRs adjacent to the TATA box, ii) the junctions contain highly conserved PBS, PPT and attachment sites, whilst an IR accurately surrounds the terminal PPT, iii) multiple identical PPTs cluster next to the 3'LTR and iv) the integrase signal occurs at the predicted domain at the 3' end of the LTRs. These features may be among the underlying factors that contribute towards the Sirevirus distribution in plants.

\section{The high conservation of the Sirevirus LTR junctions} indicates strict sequence requirements towards reverse transcription and integration

The PPT and the pairing of the tRNA molecule to the PBS are used as primers during reverse transcription to initiate

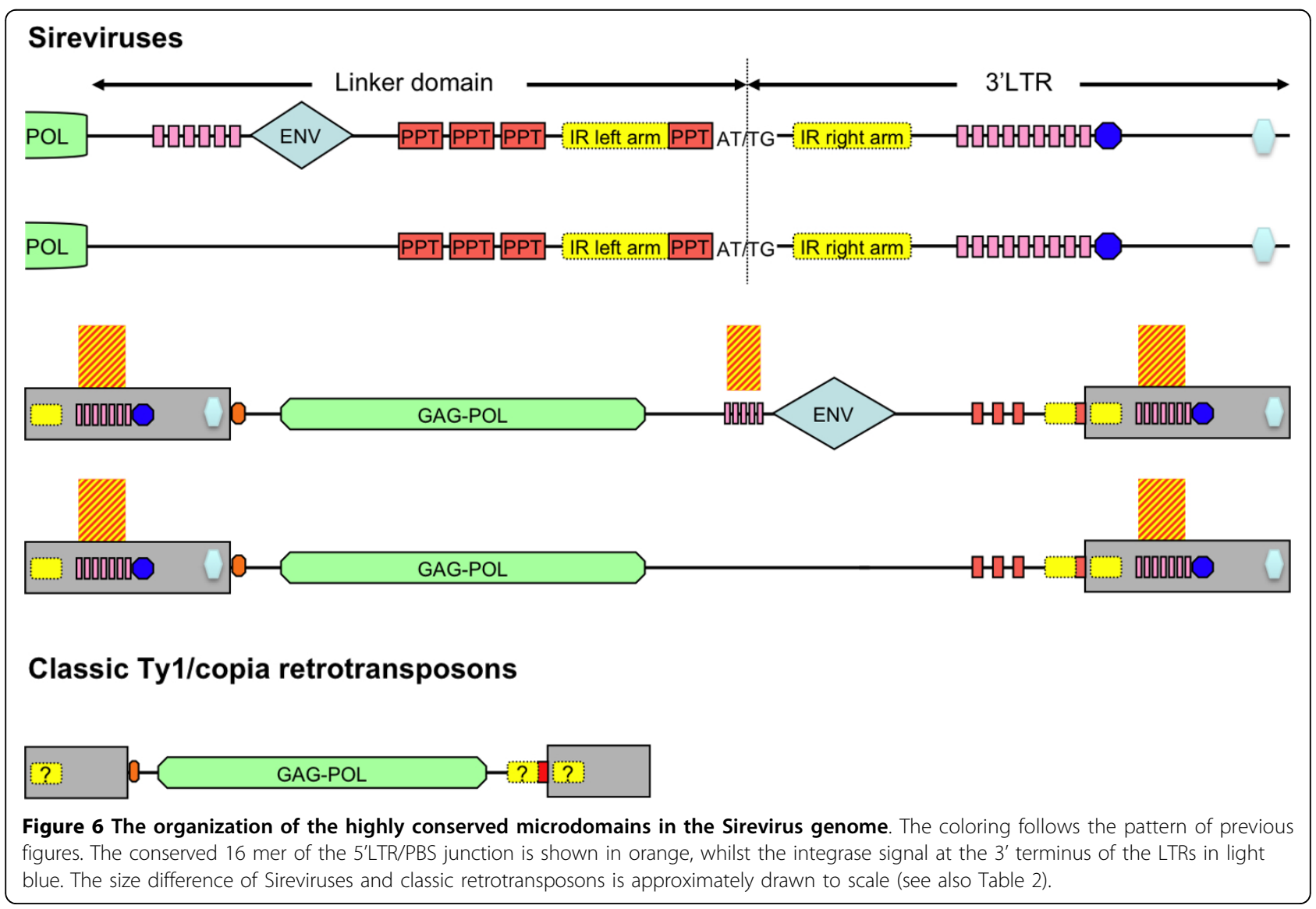


plus and minus strand cDNA synthesis, respectively. Both steps are followed by template switching events (or jumps) that transfer the partial DNA fragments to the other side of the template, so as to resume and complete cDNA synthesis [16]. During the jumps the intervening nucleotides inbetween the 5'LTR-PBS and PPT-3'LTR junctions are ordinarily added to the outer sides of the linear cDNA intermediate, hence generating the attachment sites. In the first step of integration in the new genomic location, namely the 3' end processing, INT recognizes and cleaves the intervening nucleotides from the 3 ' ends of the linear cDNA, thereby preparing the new copy for nucleophilic attack [26].

According to the TEIRESIAS algorithm the two most conserved motifs of the Sirevirus genome are the PPT/ 3'LTR and 5'LTR/PBS junctions. The extreme sequence conservation of the two domains, which appear to be very important for reverse transcription and integration [2], reflects the strict sequence requirements of the Sirevirus replication strategy. Also, the presence of the integrase signal is unique among LTR retrotransposons and suggests the tight cooperation of INT with the Sirevirus LTRs. In contrast, the respective regions of classic elements show extensive sequence variation, although a large proportion maintains a common PPT structure that relates to the Sirevirus PPT octamer. We propose that the primer for plus strand cDNA synthesis in the majority of Pseudoviridae consists of eight bases, which may tolerate some variability in classic elements, but is strictly conserved in Sireviruses.

\section{Which is the role of the multiple PPTs?}

The presence of multiple PPTs within retroelements is not unprecedented, however their role in plus strand cDNA synthesis is unclear. One extra PPT is present in the linkers of the $E N V$-containing Ty3-gypsy elements Athila4 and Calypso [35], whilst one functional PPT has been reported in the coding domains of Ty1 and HIV1 [36]. PPT-primed cDNA synthesis is a rapid event and when extra PPTs occur in the coding domain, synthesis initiates simultaneously with the natural PPT [37]. Yet, the upstream-primed cDNA must synthesize several kilobases of sequence and as a result it is outcompeted by the natural PPT-primed cDNA due to kinetic competition [36]. In Sireviruses kinetic competition is unlikely to prevent strand transfer, since the distance between the PPTs is small. The critical parameter might be the availability of the tRNA primer at the 3' end of the minus DNA strand that is utilized during the second strand transfer to provide complementarity to the PBS sequence at the 5' end of the minus DNA strand. Rapid $R H$ degradation of the tRNA after it is copied in the natural PPT-primed cDNA may prohibit strand transfer from internally initiated cDNAs [36].
The upstream PPTs also lack the correct attachment site and the IR motif, which further obscures their functionality. The transposition ability of the retroelement is dependant on the attachment site [26]. If the PPT is not followed by the correct signal for INT processing, the cDNA intermediate will be unable to insert in the new genomic location. On the other hand, the strict nucleotide integrity of the upstream PPTs, their high copy number and preferential clustering next to the 3'LTR point towards selection. They may facilitate genetic recombination between the two RNA genomes in the VLP [38], or offer a relative replication advantage to the genus [39]. In any way, based on the fact that retrotransposons with $E N V$ genes contain near their 3'LTR repeats that show similarity to PPTs, Havecker et al. (2004) proposed their involvement in reverse transcription.

\section{The IR can act as facilitator for the correct PPT primer formation}

Research in Ty1 has shown that the PPT sequence by itself is not sufficient to promote correct $R H$ processing. Additional recognition signals in the form of secondary structures near the PPT domain may be needed to affect the overall conformation of the region and attract the $R H$ for PPT formation and subsequent DNA elongation [37]. A highly conserved IR that forms a stem loop secondary structure, of which the 8 bp PPT retains its outmost left side, seems as the most suitable candidate for such a recognition signal. Presumably, this configuration preferentially 'exposes' the terminal PPT and renders it accessible to $R H$ for precise cleavage, which will automatically generate the highly conserved attachment site. Many classic Ty1/copia elements also contain the IR, which suggests that this motif may have a universal role in retrotransposon life cycle.

\section{Are the RM boxes involved in the regulation of Sirevirus activity?}

Sireviruses share highly similar versions of a novel RM, which in conjunction to the exceptional high copy number and flanking sequence divergence, make the conservation remarkable. Symmetry is a frequent feature of regulatory sequences, including box I of Tnt1 [14], an activator in the 5'UTR of Copia element [40] and the Pal elements in the $\beta$-glucokinase promoter of neuroendocrine pancreatic, gut and brain cells in humans and mice [41]. The position of the boxes in the LTRs and upstream of the $E N V$-gene does not seem random and they also form CpG islands that are by default found at the 5' side of genes regulating their expression [29]. Interestingly, Sireviruses lacking the boxes may have similar repeated structures, as large sections of their LTRs and upstream of the ENV are particularly CG-rich and generate CpG islands (data not shown). Despite these unique characteristics, we could not identify 
a common binding site targeted by a single DBF. It appears that minor variation, notably the presence of one intervening base or a substitution in the symmetrical motif, may confer different binding properties that may enable interactions with different nuclear factors. Future promoter analysis experiments could elucidate the role, if any, of the RMs in the Sirevirus activation network.

\section{Clues into the functionality of the envelope gene}

The function of the $E N V$-gene in plant retroelements has not been experimentally proven and due to the plant cell wall its role remains highly controversial [42]. So far it has been known that $E N V$-containing plant retroelements express their $E N V$ either through a spliced $E N V$-like mRNA, for example splice acceptor sites have been predicted for the Ty3-gypsy BAGY2 and Athila elements $[35,43]$, or through stop codon suppression in SIRE1 [44]. However, SIRE1 is an atypical Sirevirus, as pol and $E N V$ are separated only by a stop codon. The sequence of the Sirevirus $E N V$-gene is highly variable [18]. Peterson-Burch and Voytas (2002) hypothesized that the Sirevirus $E N V$ may be expressed from an internal promoter, although they were not able to identify promoters or transcription factors-binding sites upstream of the $E N V$. Our work indicates that specific motifs, which are also the epicentre of CpG islands, may have a regulatory role in the 5' vicinity of $E N V$, which offers support to the internal promoter hypothesis for $E N V$ expression, although we were not able to conclusively identify one. Intriguingly, CpG islands are predicted in the same domain for six $E N V$-containing Sireviruses that lack the RM boxes, even in the pol/ENV junction of SIRE1-1. Collectively, we believe our observations to be the first set of evidence concerning the Sirevirus ENV expression and their potential capacity to become virulent during their life cycle.

\section{Are Sireviruses capable of horizontal transmission?}

Sireviruses are abundant and widely dispersed in plants [19]. Moreover, it is the only Pseudoviridae genus to contain an ENV gene, which is hypothesized as being funtional. Has horizontal transmission, which is a sporadic incident, aided their proliferation? Possibly as plant viruses do, Sireviruses accumulate in the cytoplasm within their envelope and wait for a feeding invertebrate to ingest and transfer them to another plant [35]. If horizontal transmission has occurred at high rates, then one would expect to frequently encounter elements with high nucleotide similarity in diverse plant phyla, which does not seem to be the case. However, this observation does not rule out the possibility that horizontal transfer can rarely happen, but when it does occur, Sireviruses quickly colonize the new host assisted by their unique genomic organization. Alternatively, the Sirevirus envelope or gag capsid may act as a retrovirus movement protein and enable VLP transport outside the cell to infect different tissues of the same host, including meristem or germ cells, thereby enhancing the vertical transmission rate $[45,46]$.

\section{The distinctive relationship of the maize Sireviruses OPIE- 2 and PREM-2}

The conservation of the motifs is exemplified in the closely related OPIE- 2 and PREM- 2 maize elements. The alignment of their LTRs provides striking results, as the microdomains are the only highly similar segments amidst diverse sequences (Figure S5 in Additional file 2 ), often even higher than regions of pol and particularly gag. These contradict the sequence differentiation that has been reported for the Tnt1 superfamily and to the best of our knowledge this is the first time such a pattern of sequence conservation is shown. The Tnt1 superfamily consists of retrotransposons with surprisingly homologous genomes that colonize species of the Solanaceae family $[47,48]$. The only region that exemplifies high genomic variability is the LTR domain that harbors the cis-acting motifs $[49,50]$. The sequence divergence and evolution of the motifs has equipped the Tnt1 superfamily with high adaptive regulatory capacity that allowed subgroups to evolve differently and colonize new Solanaceae genomes $[51,52]$. It appears, therefore, that the OPIE-2/PREM-2 conservation pattern contradicts those of the Tnt1 superfamily and implies that the two elements may respond to the same stimuli and have the same transmission strategy.

\section{Conclusions}

Aided by the novel computational analysis which was orchestrated by the TEIRESIAS pattern discovery algorithm, we identified a plethora of highly conserved motifs in specific non-coding regions of the Sirevirus genome (Figure 6) and we hypothesized that these may be among the factors that underlie the extensive propagation of the genus in plant species. The motifs may be involved in the activation of the element and the ENVgene, but also influence the folding of the RNA and DNA strands, suggesting their interaction with gag and the pol enzymes during VLP assembly, reverse transcription and integration. Based on the extreme sequence divergence outside the coding domain, we proposed that Sireviruses form an ancient lineage, which challenges previous research that argued in favour of the young evolutionary origin of the genus. Amidst their diverse non-coding genome, strict functional pressures apparently act on the motifs to maintain their sequence integrity. The degree of conservation is astonishing, as the Sirevirus elements of our dataset reside in plant species that diverged 140-150 Mya (monocots/dicots) [53]. 
As vast sequence data become available from wholegenome sequencing projects, the findings of this work should enable the detailed analysis of how Sireviruses interact with their plant hosts and shape their genomes, towards which the fact that Sireviruses inhabit only plants may have important implications. While our knowledge on the evolutionary depth, distribution and genomic organization of Sireviruses will expand, more informed functional analysis of the herein introduced motifs will elucidate how and why the Sirevirus life cycle differs from other retrotransposons. In light of such knowledge, in the modern era of large-scale transcriptome analysis, and in possession of new evidence for the major influence of retrotransposon acticity on the cell transcriptional output (alternative promoters, noncoding RNAs, bidirectional transcription) [54,55], we should be able to move closer to elucidating the role of Sireviruses in the epigenetic regulation of plant genes [56]. Finally, based on recent models that propose transposable elements as being major suppliers of cis-regulatory elements to host genomes [57], Sireviruses with their novel type of RM may have been involved in the evolution of plant gene regulatory networks.

\section{Methods}

\section{Ty1/copia datasets}

The Sirevirus and classic retrotransposon datasets were constructed by retrieving sequences from various sources. The information for each element, including the source, host species and Pseudoviridae genus is provided (Table S1 in Additional file 1). The Pseudoviridae classification according to the ICTV database can be found at http://www.ncbi.nlm.nih.gov/ICTVdb/ICTVdB/ 00.097.htm. The raw sequence data (where all the conserved motifs are highlighted) of our Sirevirus database is available at http://bat.ina.certh.gr/downloads/Sirevirus_sequence_data.doc.

\section{Sequence analysis tools}

Multiple alignments were carried out using ClustalW at default parameters [58] and the Jalview multiple alignment editor [59] from the EBI website http://www.ebi. ac.uk/. MEGA4 [60] was used to construct the phylogenetic tree using the neighbor-joining distance method [61]. Bootstrap test of phylogeny was calculated based on 1000 replicate trees and the evolutionary distances were computed using the Poisson correction model [62]. The $R T-R H$ peptide alignment used for the analysis is provided (Additional file 4). Identification of the RM boxes and the IR were aided by the Tandem Repeat Finder [63] and Inverted Repeat Finder algorithms [64], respectively, available at the Laboratory for Biocomputing and Informatics http://tandem.bu.edu/tools.html. The RMs were compared against known plant cis-acting regulatory sequences in the PLACE database $[27,65]$, in order to identify target sites of DBFs. Finally, CpG islands were predicted using the CpGplot package [66] from the EBI website.

\section{TEIRESIAS pattern discovery algorithm}

Discovery of sequence motifs was performed with the use of the TEIRESIAS algorithm [25]. Importantly, TEIRESIAS is guaranteed to report all maximal patterns (i.e. patterns that cannot be made more specific and still keep on appearing at the exact same positions within the input sequences, or else non-redundant patterns) meeting the structural restrictions set by the user through parameters. In other words, no heuristics are employed in the discovery process. The complete set of parameters of TEIRESIAS used in this analysis is as follows: nucleotides in the pattern $(-1)$, number of overlapping characters in the convolved pattern $(-\mathrm{c})$, maximum length of an elementary pattern $(-\mathrm{w})$, minimum number of appearances of the pattern (-k), flag for the support $k$ to be the minimum number of sequences in which a pattern should appear (compared to the minimum number of instances of the pattern) $(-v)$. Several sets of values for $\mathrm{l}, \mathrm{w}$, and $\mathrm{k}$ were used to obtain a comprehensive picture of the conservation of motifs in the input and this information was used critically and in context to produce the final observations.

Additional file 1: The Pseudoviridae dataset used in this analysis.

This file contains supplementary Table S1 showing the source, accession number, host species and Pseudoviridae genus for each retrotransposon. Click here for file

[http://www.biomedcentral.com/content/supplementary/1471-2164-1189-S1.PDF ]

Additional file 2: Phylogenetic and structural domain analysis of the Sirevirus and classic Ty1/copia retrotransposons. This file contains supplementary Figure S1 showing the phylogenetic analysis of the Ty1/copia retrotransposons based on the RT/RH domains,

supplementary Figures S2 and S3 with alignments of the IR arms of each Sirevirus and classic retrotransposon respectively, supplementary Figure S4 showing the distribution of the novel Sirevirus RM boxes, and supplementary Figure S5 with the 5'LTR alignment of the OPIE-2 and PREM-2 Sireviruses.

Click here for file

[http://www.biomedcentral.com/content/supplementary/1471-2164-1189-S2.PDF ]

Additional file 3: The stress-related DNA binding factors that target core sites within the RMs. This file contains supplementary Table S2 with information related to the binding site and its orientation within each RM and the stress nature of the DBFs.

Click here for file

[http://www.biomedcentral.com/content/supplementary/1471-2164-1189-S3.PDF ]

Additional file 4: List of the $R T / R H$ peptide sequences that were used for the construction of the Ty1/copia phylogenetic tree. Click here for file

[http://www.biomedcentral.com/content/supplementary/1471-2164-1189-S4.PDF ] 


\section{Abbreviations}

PPT: polypurine tract; LTR: long terminal repeat; ENV: envelope; VLP: virus-like particle; AP: aspartic protease; INT: integrase; RT: reverse transcriptase; $\mathrm{RH}$ : RNaseH; DBF: DNA binding factor; RM: repeated motif; UTR: untranslated region; PBS: primer binding site; ICTV: International Committee on the Taxonomy of Viruses; ORF: open reading frame; IR: inverted repeat.

\section{Acknowledgements}

We thank T. Thedosiou, T. Schwarzacher, E. Simeonidis and V. Apostolidou for reading and improving this manuscript. $A B$ and ND were supported by the BioSapiens Network of Excellence (contract number LSHG-CT-2003503265).

\section{Author details}

'Department of Biology and Environmental Science, School of Life Sciences, University of Sussex, Brighton, UK. ${ }^{2}$ Institute of Agrobiotechnology, Centre for Research and Technology Hellas, Thessaloniki 57001, Greece. ${ }^{3}$ Department of Genetics and Plant Breeding, Aristotle University of Thessaloniki AUTH, Thessaloniki 54006, Greece.

\section{Authors' contributions}

$A B$ conceived and designed the study, carried out the data collection, analysis, and interpretation, and drafted the manuscript. ND carried out pattern discovery with the TEIRESIAS algorithm, assisted in data interpretation and critically revised the manuscript. AT assisted in data interpretation and critically revised the manuscript. SP conceived and designed the study, and critically revised the manuscript. All authors read and approved the final manuscript.

Received: 11 November 2009

Accepted: 4 February 2010 Published: 4 February 2010

\section{References}

1. Hansen C, Heslop-Harrison JS: Sequences and phylogenies of plant pararetroviruses, viruses, and transposable elements. Advances in Botanical Research Incorporating Advances in Plant Pathology 2004, 41:165-193.

2. Kumar A, Bennetzen JL: Plant retrotransposons. Annu Rev Genet 1999, 33:479-532.

3. Bousios A, Saldana-Oyarzabal I, Valenzuela-Zapata AG, Wood C, Pearce SR: Isolation and characterization of Ty1-copia retrotransposon sequences in the blue agave (Agave tequilana Weber var. azul) and their development as SSAP markers for phylogenetic analysis. Plant Science 2007, 172(2):291-298

4. Feschotte C, Jiang N, Wessler SR: Plant transposable elements: Where genetics meets genomics. Nature Reviews Genetics 2002, 3(5):329-341.

5. Pearce SR, Harrison G, HeslopHarrison PJS, Flavell AJ, Kumar A: Characterization and genomic organization of Ty1-copia group retrotransposons in rye (Secale cereale). Genome 1997, 40(5):617-625.

6. Vitte C, Panaud O: LTR retrotransposons and flowering plant genome size: emergence of the increase/decrease model. Cytogenetic and Genome Research 2005, 110(1-4):91-107.

7. Pereira $\mathrm{V}$ : Insertion bias and purifying selection of retrotransposons in the Arabidopsis thaliana genome. Genome Biol 2004, 5(10):R79.

8. McCarthy EM, Liu J, Lizhi G, McDonald JF: Long terminal repeat retrotransposons of Oryza sativa. Genome Biol 2002, 3(10):RESEARCH0053.

9. Baucom RS, Estill JC, Chaparro C, Upshaw N, Jogi A, Deragon JM, Westerman RP, SanMiguel PJ, Bennetzen JL: Exceptional Diversity, NonRandom Distribution, and Rapid Evolution of Retroelements in the B73 Maize Genome. Plos Genetics 2009, 5(11):e1000732.

10. Schnable PS, Ware D, Fulton RS, Stein JC, Wei FS, Pasternak S, Liang CZ, Zhang JW, Fulton L, Graves TA, Minx P, Reily AD, Courtney L, Kruchowski SS, Tomlinson C, Strong C, Delehaunty K, Fronick C, Courtney B, Rock SM, Belter E, Du FY, Kim K, Abbott RM, Cotton M, Levy A, Marchetto P, Ochoa K, Jackson SM, Gillam B, et al: The B73 Maize Genome: Complexity, Diversity, and Dynamics. Science 2009, 326(5956):1112-1115.

11. Vicient CM, Suoniemi A, Anamthamat-Jonsson K, Tanskanen J, Beharav A, Nevo E, Schulman AH: Retrotransposon BARE-1 and its role in genome evolution in the genus Hordeum. Plant Cell 1999, 11(9):1769-1784.

12. Wicker T, Sabot F, Hua-Van A, Bennetzen JL, Capy P, Chalhoub B, Flavell A, Leroy $\mathrm{P}$, Morgante M, Panaud O, Paux E, SanMiguel P, Schulman AH: A unified classification system for eukaryotic transposable elements. Nature Reviews Genetics 2007, 8(12):973-982.

13. Peterson-Burch BD, Voytas DF: Genes of the Pseudoviridae (Ty1/copia retrotransposons). Mol Biol Evol 2002, 19(11):1832-1845.

14. Grandbastien MA, Lucas H, Morel JB, Mhiri C, Vernhettes S, Casacuberta JM: The expression of the tobacco Tnt1 retrotransposon is linked to plant defense responses. Genetica 1997, 100(1-3):241-252.

15. Vernhettes $S$, Grandbastien MA, Casacuberta JM: In vivo characterization of transcriptional regulatory sequences involved in the defence-associated expression of the tobacco retrotransposon Tnt1. Plant Molecular Biology 1997, 35(5):673-679.

16. Wilhelm M, Wilhelm FX: Reverse transcription of retroviruses and LTR retrotransposons. Cellular and Molecular Life Sciences 2001, 58(9):1246-1262.

17. Boeke JD, Eickbush TH, Sandmeyer SB, Voytas DF: Index of Viruses Pseudoviridae (2006). ICTVdB - The Universal Virus Database, version 4 Columbia University, New York, USABuchen-Osmond C 2006.

18. Havecker ER, Gao X, Voytas DF: The sireviruses, a plant-specific lineage of the Ty1/copia retrotransposons, interact with a family of proteins related to dynein light chain. Plant Physiol 2005, 139(2):857-868.

19. Havecker ER, Gao X, Voytas DF: The diversity of LTR retrotransposons. Genome Biol 2004, 5(6):225

20. Gao X, Havecker ER, Baranov PV, Atkins JF, Voytas DF: Translational recoding signals between gag and pol in diverse LTR retrotransposons. RNA-Publ RNA Soc 2003, 9(12):1422-1430.

21. Nagaki K, Song JQ, Stupar RM, Parokonny AS, Yuan QP, Ouyang S, Liu J, Hsiao J, Jones KM, Dawe RK, Buell CR, Jiang JM: Molecular and cytological analyses of large tracks of centromeric DNA reveal the structure and evolutionary dynamics of maize centromeres. Genetics 2003, 163(2):759-770.

22. Holligan $D$, Zhang $X Y$, Jiang $N$, Pritham EJ, Wessler SR: The transposable element landscape of the model legume Lotus japonicus. Genetics 2006 174(4):2215-2228.

23. Kenward KD, Bai D, Ban MR, Brandle JE: Isolation and characterization of Tnd-1, a retrotransposon marker linked to black root rot resistance in tobacco. Theor Appl Genet 1999, 98(3-4):387-395.

24. Xiong $Y$, Eickbush TH: Origin and Evolution of Retroelements Based Upon Their Reverse-Transcriptase Sequences. Embo J 1990, 9(10):3353-3362.

25. Rigoutsos I, Floratos A: Combinatorial pattern discovery in biological sequences: the TEIRESIAS algorithm. Bioinformatics 1998, 14(1):55-67.

26. Goff SP: Genetics of Retroviral Integration. Annu Rev Genet 1992, 26:527-544.

27. Higo K, Ugawa $Y$, Iwamoto $M$, Korenaga T: Plant cis-acting regulatory DNA elements (PLACE) database: 1999. Nucleic Acids Res 1999, 27(1):297-300.

28. Gardiner-Garden M, Frommer M: Cpg Islands in Vertebrate Genomes. Journal of Molecular Biology 1987, 196(2):261-282.

29. Ashikawa I: Gene-associated CpG islands in plants as revealed by analyses of genomic sequences. Plant J 2001, 26(6):617-625.

30. Ashikawa I, Numa H, Sakata K: Segmental distribution of genes harboring a CpG island-like region on rice chromosomes. Molecular Genetics and Genomics 2006, 275(1):18-25.

31. Grace ML, Chandrasekharan MB, Hall TC, Crowe AJ: Sequence and spacing of TATA box elements are critical for accurate initiation from the betaphaseolin promoter. J Biol Chem 2004, 279(9):8102-8110.

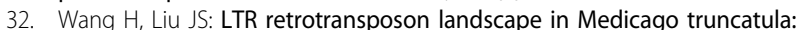
more rapid removal than in rice. BMC Genomics 2008, 9.

33. Bugreev DV, Baranova S, Zakharova OD, Parissi V, Desjobert C, Sottofattori $E_{1}$ Balbi A, Litvak S, Tarrago-Litvak L, Nevinsky GA: Dynamic, thermodynamic, and kinetic basis for recognition and transformation of DNA by human immunodeficiency virus type 1 integrase. Biochemistry 2003, 42(30):9235-9247.

34. Wicker T, Keller B: Genome-wide comparative analysis of copia retrotransposons in Triticeae, rice, and Arabidopsis reveals conserved ancient evolutionary lineages and distinct dynamics of individual copia families. Genome Research 2007, 17:1072-1081.

35. Wright DA, Voytas DF: Athila4 of Arabidopsis and Calypso of soybean define a lineage of endogenous plant retroviruses. Genome Research 2002, 12(1):122-131.

36. Bowman EH, Pathak VK, Hu WS: Efficient initiation and strand transfer of polypurine tract-primed plus-strand DNA prevent strand transfer of internally initiated plus-strand DNAs. Journal of Virology 1996, 70(3):1687-1694 
37. Heyman T, Agoutin B, Friant S, Wilhelm FX, Wilhelm ML: Plus-Strand DNA Synthesis of the Yeast Retrotransposon Ty1 Is Initiated at 2 Sites, Ppt1 Next to the 3'-Ltr and Ppt2 within the Pol Gene - Ppt1 Is Sufficient for Ty1 Transposition. Journal of Molecular Biology 1995, 253(2):291-303.

38. Friant $\mathrm{S}$, Heyman $T$, Wilhelm FX, Wilhelm M: Role of RNA primers in initiation of minus-strand and plus-strand DNA synthesis of the yeast retrotransposon Ty1. Biochimie 1996, 78(7):674-680.

39. Charneau P, Alizon M, Clavel F: A 2nd Origin of DNA Plus-Strand Synthesis Is Required for Optimal Human-Immunodeficiency-Virus Replication. Journal of Virology 1992, 66(5):2814-2820.

40. Wilson S, Matyunina LV, McDonald JF: An enhancer-region within the copia untranslated leader contains binding sites for Drosophila regulatory proteins. Gene 1998, 209(1-2):239-246.

41. Moates JM, Magnuson MA: The Pal elements in the upstream glucokinase promoter exhibit dyad symmetry and display cell-specific enhancer activity when multimerised. Diabetologia 2004, 47(9):1632-1640.

42. Peterson-Burch BD, Wright DA, Laten HM, Voytas DF: Retroviruses in plants?. Trends in Genetics 2000, 16(4):151-152

43. Vicient $\mathrm{CM}$, Kalendar R, Schulman AH: Envelope-class retrovirus-like elements are widespread, transcribed and spliced, and insertionally polymorphic in plants. Genome Research 2001, 11(12):2041-2049.

44. Havecker ER, Voytas DE: The soybean retroelement SIRE1 uses stop codon suppression to express its envelope-like protein. EMBO Rep 2003 4(3):274-277.

45. Brill LM, Nunn RS, Kahn TW, Yeager M, Beachy RN: Recombinant tobacco mosaic virus movement protein is an RNA-binding, alpha-helical membrane protein. Proc Natl Acad Sci USA 2000, 97(13):7112-7117.

46. Melcher U: The ' $30 \mathrm{~K}$ ' superfamily of viral movement proteins. Journal of General Virology 2000, 81:257-266.

47. Casacuberta JM, Vernhettes S, Audeon C, Grandbastien MA: Quasispecies in retrotransposons: a role for sequence variability in Tnt1 evolution. Genetica 1997, 100(1-3):109-117.

48. Manetti ME, Rossi M, Costa APP, Clausen AM, Van Sluys MA: Radiation of the Tnt1 retrotransposon superfamily in three Solanaceae genera. BMC Evol Biol 2007, 7.

49. Beguiristain T, Grandbastien MA, Puigdomenech P, Casacuberta JM: Three Tnt1 subfamilies show different stress-associated patterns of expression in tobacco. Consequences for retrotransposon control and evolution in plants. Plant Physiol 2001, 127(1):212-221.

50. Costa APP, Scortecci KC, Hashimoto RY, Araujo PG, Grandbastien MA, Van Sluys MA: Retrolyc1-1, a member of the Tnt1 retrotransposon superfamily in the Lycopersicon peruvianum genome. Genetica 1999, 107(13):65-72.

51. Araujo PG, Casacuberta JM, Costa APP, Hashimoto RY, Grandbastien MA, Van Sluys MA: Retrolyc1 subfamilies defined by different U3 LTR regulatory regions in the Lycopersicon genus. Molecular Genetics and Genomics 2001, 266(1):35-41.

52. Vernhettes S, Grandbastien MA, Casacuberta JM: The evolutionary analysis of the Tnt1 retrotransposon in Nicotiana species reveals the high variability of its regulatory sequences. Mol Biol Evol 1998, 15(7):827-836.

53. Chaw SM, Chang CC, Chen HL, Li WH: Dating the monocot-dicot divergence and the origin of core eudicots using whole chloroplast genomes. J Mol Evol 2004, 58(4):424-441.

54. Faulkner GJ, Kimura Y, Daub CO, Wani S, Plessy C, Irvine KM, Schroder K, Cloonan N, Steptoe AL, Lassmann T, Waki K, Hornig N, Arakawa T, Takahashi H, Kawai J, Forrest ARR, Suzuki H, Hayashizaki Y, Hume DA, Orlando V, Grimmond SM, Carninci P: The regulated retrotransposon transcriptome of mammalian cells. Nature Genetics 2009, 41(5):563-571.

55. Mercer TR, Dinger ME, Mattick JS: Long non-coding RNAs: insights into functions. Nature Reviews Genetics 2009, 10(3):155-159.

56. Slotkin RK, Martienssen R: Transposable elements and the epigenetic regulation of the genome. Nature Reviews Genetics 2007, 8(4):272-285.

57. Feschotte C: Transposable elements and the evolution of regulatory networks. Nature Reviews Genetics 2008, 9(5):397-405.

58. Chenna R, Sugawara H, Koike T, Lopez R, Gibson TJ, Higgins DG, Thompson JD: Multiple sequence alignment with the Clustal series of programs. Nucleic Acids Res 2003, 31(13):3497-3500.

59. Waterhouse AM, Procter JB, Martin DMA, Clamp M, Barton GJ: Jalview Version 2-a multiple sequence alignment editor and analysis workbench. Bioinformatics 2009, 25(9):1189-1191.
60. Tamura K, Dudley J, Nei M, Kumar S: MEGA4: Molecular evolutionary genetics analysis (MEGA) software version 4.0. Mol Biol Evol 2007, 24(8):1596-1599.

61. Saitou N, Nei M: The Neighbor-Joining Method - a New Method for Reconstructing Phylogenetic Trees. Mol Biol Evol 1987, 4(4):406-425.

62. Zuckerkandl E, Pauling L: Evolutionary divergence and convergence in proteins. Evolving Genes and Proteins Academic Press, New YorkBryson V, Vogel HJ 1965, 97-166.

63. Benson G: Tandem repeats finder: a program to analyze DNA sequences. Nucleic Acids Res 1999, 27(2):573-580.

64. Gelfand Y, Rodriguez A, Benson G: TRDB - The Tandem Repeats Database. Nucleic Acids Res 2007, 35:D80-D87.

65. Prestridge DS: SIGNAL SCAN - A COMPUTER-PROGRAM THAT SCANS DNA-SEQUENCES FOR EUKARYOTIC TRANSCRIPTIONAL ELEMENTS. Computer Applications in the Biosciences 1991, 7(2):203-206.

66. Larsen F, Gundersen G, Lopez R, Prydz H: Cpg Islands as Gene Markers in the Human Genome. Genomics 1992, 13(4):1095-1107.

doi:10.1186/1471-2164-11-89

Cite this article as: Bousios et al:: Highly conserved motifs in non-coding regions of Sirevirus retrotransposons: the key for their pattern of distribution within and across plants?. BMC Genomics 2010 11:89.

\section{Submit your next manuscript to BioMed Central and take full advantage of:}

- Convenient online submission

- Thorough peer review

- No space constraints or color figure charges

- Immediate publication on acceptance

- Inclusion in PubMed, CAS, Scopus and Google Scholar

- Research which is freely available for redistribution

Submit your manuscript at www.biomedcentral com/submit
C) Biomed Central 